\title{
Bayesian networks Applications on Dependability, Risk Analysis and Maintenance
}

\author{
G. Medina Oliva, P. Weber, C. Simon, B. Iung \\ CRAN, Nancy-Université, CNRS, Boulevard des Aiguillettes B.P. 70239 F-54506 Vandœuvre lès Nancy (e-mail: \\ \{gabriela.medina-oliva, benoit.iung\}@cran.uhp-nancy.fr,\{philippe.weber, christophe.simon\}@esstin.uhp-nancy.fr
}

\begin{abstract}
In this paper, a bibliographical review is presented about the use of Bayesian networks over the last decade on dependability, risk analysis and maintenance. It is shown an increasing trend of the literature and of the application of Bayesian networks in fields related to reliability, safety and maintenance. This trend is due to the benefits that Bayesian networks provide in contrast with other classical methods of dependability analysis such as Markov Chains and Fault Trees. Some of these benefits are: to model and to analyze complex systems, to make predictions as well as diagnostics, to compute exactly the occurrence probability of an event, to update the calculations according to evidences and to represent multimodal variables. This review is based on an extraction of 200 references; the most representative are presented.
\end{abstract}

Keywords: Bayesian networks dependability, risk analysis, maintenance.

\section{INTRODUCTION}

The management of complex industrial systems requires a high performance and a reliability analysis. Nowadays, some of the problems in the reliability field are: the representation and modeling of the system, the quantification of system model parameters and the representation, propagation and quantification of the uncertainty in the system behavior (Zio, 2009). Other important issues to be considered in our domains of interest are: the temporal aspect in the reliability and risk analysis, the integration of qualitative information with quantitative knowledge on the different abstraction levels, the nature of multi-state elements and the dependences between events.

Some papers such as Mahadevan (2001), Boudali and Dugan (2005b), Langseth and Portinale (2007), Bayraktarli et al. (2005) show the increasing interest on the use of Bayesian Networks to estimate and to improve reliability and safety of systems over the last decade. Therefore, we propose to analyze the evolution of literature about Bayesian Networks and their applications on dependability, risk analysis and maintenance. For this purpose, we build a database of references from 1990 to 2008 with different bibliographical research tools (google scholar). In this paper, the most relevant articles according to their citation number were cited.

\section{BAYESIAN NETWORKS}

The Bayesian Networks (BN) are a modeling probabilistic formalism that allows a robust and efficient framework for reasoning with uncertain knowledge. $\mathrm{A} \mathrm{BN}$ is a directed acyclic graph (DAG) in which the nodes represent the system variables and the arcs symbolize the dependencies or the cause-effect relationships among the variables. BN perform the variables joint distribution factorization based on the conditional dependencies. The main objective of $\mathrm{BN}$ is to calculate the distribution probabilities in a group of variables.
The principles of this modeling tool are explained by Jensen, 1996 and Pearl, 1988.

The BNs characteristics are:

- a group of nodes and a group of directed arcs,

- a table of prior probabilities $\mathrm{P}(\mathrm{X})$ associated to the parent node $\mathrm{X}$,

- a table of conditional probabilities $\mathrm{P}(\mathrm{Y} / \mathrm{X})$ attached to the node $\mathrm{Y}$ whose father is $\mathrm{X}$. It defines the probability distributions over the states of $\mathrm{Y}$ given the states $\mathrm{X}$.

In order to provide a reliability global estimation, BNs permit to merge knowledge of diverse natures in one model: data from feedback experience, experts' judgment (express through logical rules, equations or subjective probabilities), analyze the behavior of the studied system (functional and dysfunctional analysis) and observations. Usually, to build the model, it is necessary to use these sources of information. However, there is few feedback experience data particularly in the domains of dependability, risk analysis and maintenance, for this reason the research work utilized mainly the experts' judgment to build the structure of the models (Bouissou \& Nguyen, 2002).

A general inference mechanism (that permits the prevision as well as the diagnostic) is used to collect and incorporate the new information (evidences) gathered in a study. The Bayes' theorem also allows updating a group of events' probabilities according to the observed facts and the BN structure.

\section{LITERATURE OF BN APPLICATION ON DEPENDABILITY, RISK ANALYSIS AND MAINTENANCE}

In the specialized literature about BNs, most of the references are about improving the learning and inference algorithms. Nonetheless, we have built a database containing 200 articles about BNs' application on dependability, risk analysis and maintenance which shows a continual increment on the number of references and a scientific and industrial interest in 
this tool. The first contributions were done by (Castillo et al., 1997), (Torres-Toledano \& Sucar, 1998), (Arroyo et al, 1998), (Kang \& Golay, 1999). Figure 1 shows that most of the references are about dependability with $64 \%$ of the publications, risk analysis with $23 \%$ and maintenance with $13 \%$.

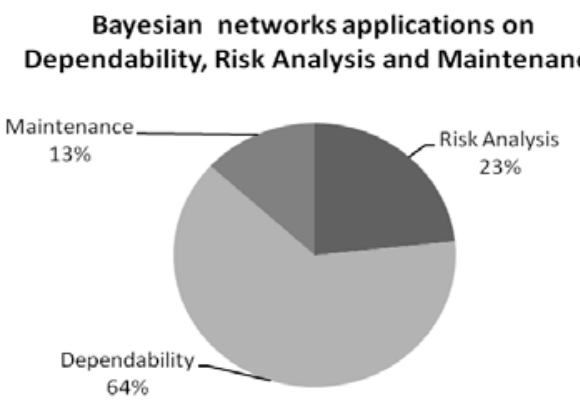

Figure 1. Distribution according to the domain dependability, risk analysis and maintenance

\subsection{Application in dependability}

BNs are more and more utilized for the dependability analyses, concerning aspects like safety, reliability, availability and maintainability. Figure 2 shows the number of articles per year relating to the BNs application on dependability analyses. Since the year 2000, we observe an important rising of $800 \%$ on its application due to the modeling benefits that BNs can offer.

The aim of dependability is to provide a prediction of a parameter (remaining time to fail, mean time to failure, etc) which is an input data in a decision problem (for example maintenance optimization).

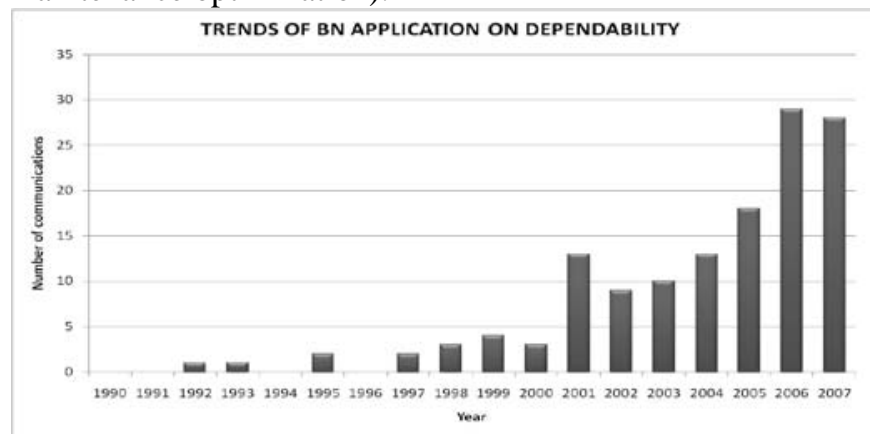

Figure 2. Bayesian network application on dependability.

The original work' objectives by (Torres-Toledano \& Sucar, 1998), (Arroyo et al., 1998) were: a) to estimate a system reliability including possibilities of failures' dependences and; b) to model complex systems. This work has also compared classical dependability methods with BNs: (Mahadevan et al., 2001), (Bobbio et al., 2001), (Bobbio et al., 2003).

The project BOLARR emphasizes dynamic modeling for risk analyses (Welch, 2001) through Bayesian Networks and, in parallel, the project SERENE's aims at formalizing the experts' reasoning in order to evaluate the different aspects of dependability on critical systems (Bouissou et al., 1999).With the objective to provide a model with several abstraction levels, the project SERENE is also based on building a hierarchical object oriented $\mathrm{BN}$ with the scope to incorporate the factors that influence the system dependability.

With reference to software reliability area, $\mathrm{BN}$ modeling has been also valuable in order to support the processes of software developments (De Melo \& Sanchez, 2008). There are some significant work in which the objective is to assess a reliability prediction within a software taking into account the operational conditions (Bai, 2005), (Bai et al., 2005). Axel and Helminen (2001) present how a BN, for a software safety standard, can be merged with a $\mathrm{BN}$ on the reliability estimation of software based on digital systems.

Wilson \& Huzurbazar (2006) describe different application contexts of $\mathrm{BN}$ in the reliability field: known or unknown conditional probabilities, taking into account new data in order to improve the conditional probabilities estimation. Helminen \& Pulkkinen (2003) exploit the BN abilities when combining experts' judgments with the feedback experience data to estimate the reliability of a motor protection critical system.

At last, Langseth \& Portinale (2007) wrote a summary about different building steps in a BN and the use of this formalism in reliability.

To conclude, an overview of the modeling aspects to be considered in dependability analysis is to:

a. include the temporal aspect in the reliability analysis,

b.consider exogenous variables to represent maintenance action events, production level and environment conditions,

c. integrate, in one model, the technical, organizational, informational, decisional and human aspects and the impacts on the system's functioning,

d. consider multi-state elements,

e. consider dependences between events,

f. characterize, represent and propagate epistemic uncertainties in reliability analysis of complex systems.

BNs provide efficient modeling solutions for the points $a$ and $d$ (Boudali \& Dugan, 2005b), (Weber \& Jouffe, 2006) (Montani et al., 2006), $b$ (Weber et al., 2004), e (TorresToledano \& Sucar, 1998), (Arroyo et al., 1998), $f$ (Simon et al, 2009) but $c$ has not been actually covered.

The recent work interests are the reliability estimation including the temporal aspect by the use of Dynamic Bayesian Networks (Boudali \& Dugan, 2005b), (Weber \& Jouffe, 2006). Montani et al., (2006) worked on the integration of the dynamic aspect by the transformation of dynamic fault trees into dynamic BNs. Dynamic Bayesian Networks models are able to represent the impacts on system reliability of the operational conditions (representing the maintenance action events, production level, environment conditions) by means of exogenous variables (Weber et al., 2004). 
To estimate the reliability of a system, Neil et al. (2007) built hybrid BNs adding discrete and continuous nodes. The algorithm combines a dynamic time sampling to the classical propagation algorithms. The time sampling of the continuous variables is updated by taking into account the evidence. The authors present this concept as an alternative method to simulation methods such as Markov Chain Monte Carlo (MCMC).

\subsection{Application in risk analysis}

The risk evaluation needs a systematic research of accidental scenarios, including the component and the operator behavior. The objective of these analyses is to evaluate the failures' event frequency as well as their consequences. They provide the elements that help decision making in terms of design evolution, operation, preparation and risk management.

Since 2001, BNs have been used to analyze these risky situations. Particularly, BNs represent a formalism use in the risk analyses domain due to their capacity to deal with probabilistic data and to model the dependencies between events. Figure 3 shows the development of BN research focused on risk analysis. From 2001 to 2007, the number of references per year has been multiplied by 4 .

The first contributions on this field were made by Hudson et $\mathrm{al}$, (2001), where they use BN as a key component of a decision support system for assessing terrorist threats against military installations. Gulvanessian and Holicky (2001) use a BN to analyze the efficiency of fire protection systems, and to find the most effective arrangements in real situations. Hansen and Simonsen (2002) show that BNs are used to establish a realistic model of the causation probability (failure rates) in an integrated software package for grounding and collision analysis.

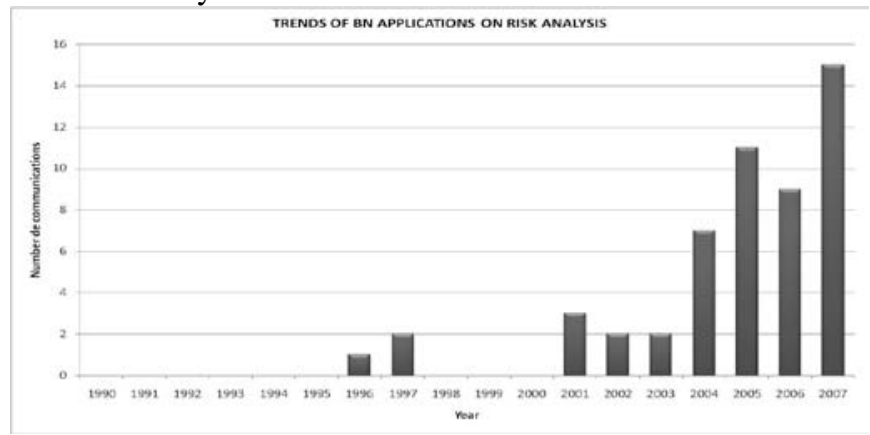

Figure 3. Bayesian Network application on risk analysis.

In 2002, Embrey (2002) takes into account human factors by using influence diagrams to analyze and to anticipate critical systems' failure. Then, Kim \& Seong (2006) describe a BN model including human factors which objective is to evaluate the effects of several scenarios in nuclear industry. The same authors use BN to observe the influencing factors in human reliability (Kim et al., 2006).

Other important contributions were made by Cornalbaa and Giudicib (2004) who present a work in which BN approach is used in order to develop a statistical model to measure and, consequently, predict, the operational risks to which a banking organization is subject. Bayraktarli et al. (2005) work with the application of $\mathrm{BN}$ for earthquake risk management. It is found that the uncertainties associated with all elements in the functional chain of an earthquake from the source mechanism, site effects, structural response, damage assessments and consequence assessment can be handled consistently using BN. Straub (2005) demonstrates the advantages of $\mathrm{BN}$ for the application in risk assessments for natural hazards. Lee and Lee (2006) propose a quantitative assessment framework integrating the inference process of $\mathrm{BN}$ to the traditional probabilistic risk analysis in order to consider the effects predicted from an evolution of environmental conditions of waste disposal facilities.

In the maritime field, some work aims at developing BN approaches to consider the human and organizational factors in a risk analysis. Norrington et al. (2007) describe the process of the experts' judgments to build a BN. A significant BN approach was developed by Trucco et al. (2008) which demonstrates the correlation between sources events of a collision accident.

In risk analyses, the recent publications of Leger et al. (2008) and Duval et al. (2007) propose a BN modeling by structuring the model in different levels: organization/ actions/ technique. The aim of these works is to quantitatively estimate the risk relating to an industrial system operation (occurrence probability of scenarios) and the evaluation of technical, human and organizational barriers' impact on the global system performance. The originality of these models is the unification formalism, based on BN, of functional, dysfunctional, behavioral and organizational knowledge of a system.

Thus, as highlighted before, in order to perform an efficient risk analysis it is necessary to:

a. model the dependencies between events,

b. demonstrate the correlation between sources events of an accident,

c. quantitatively estimate the risk relating to an industrial system operation and to evaluate the technical, human and organizational aspects barriers' impact on the system global performance,

d. integrate qualitative (functional, dysfunctional and organizational analysis) information with quantitative knowledge (technical and financial levels) on the different abstraction levels,

e. consider all feasible accidents or failure scenarios and their related consequences, with the occurrence probability of such scenarios,

f. search and model causal links among the system elements (components, structures, human, etc) and to integrate their behaviour so as to develop quantification for the system as a whole,

g. model the relationship between the context (environment and operational conditions) and the probability of human and technical failures,

h. characterize, represent and propagate epistemic uncertainties. 
i. take into account the resilient aspect of human operators and organizations,

j. include the temporal aspect in the risk analysis.

In this field, BNs enables to perform effective risk analyses fulfilling the following needs: $a, c, d, f$ and $g$ Leger et al. (2006), (2008) and Duval et al. (2007), $b$ Trucco et al. (2008), $e$ Kim \& Seong (2006) and Kim et al., 2006, nonetheless some of the pending issues in this domain are $h, i$ and $j$.

\subsection{Application in maintenance}

The BNs are used in work concerning maintenance decision and performances' evaluation. Figure 4 illustrates the increasing use of this tool. In 1999, it is observed the beginning of research activities which are increasing with a factor of 3 between 2000 and 2007. The activities in this field are recent so, there are few references.

In maintenance Kang \& Golay (1999) proposed a model with influence diagrams which consider evidences. The purpose is to estimate the future state of a system after a particular action. The proposal of an action is made based on the conditional probabilities and the utility values. TRENDS OF BN APPLICATION ON MAINTENANCE

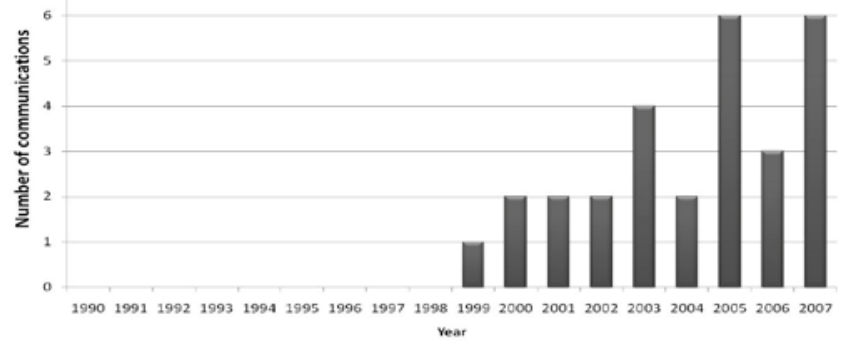

Figure 4. Bayesian network application on maintenance.

The performances' analyses of a system and modeling the prognostic process are the key points for maintenance optimization. The BN model developed by Weber et al. (2001), are built including the functional and dysfunctional analysis of the system, allowing to its global performance estimation (Muller et al., 2008).

Based on the papers (Weber and Jouffe, 2006) and (Iung et al., 2005), the causal relationships between degradation/ cause/ consequence are modelled in a dynamic bayesian network (DBN). Moreover, utility nodes are integrated into the probabilistic model.

In relation to the modeling of a real maintenance problem, Celeux et al. (2006) propose a questioning procedure for the experts' judgment. This procedure is set up by rules to collect the information and to build the network structure. The model's parameters are determined by feedback experience data and later by expertise.

To optimize maintenance strategies, a complete study should be performed, including the following aspects: a. to model the functional and dysfunctional analysis of the system, allowing its global performance estimation,

b. to model the causal relationships between degradation/ cause/ consequence,

c. to integrate qualitative (functional, dysfunctional and organizational analysis) information with quantitative knowledge (technical and financial level) on the different abstraction levels,

d. to model the degradation mechanisms and to represent: the influence factors (service time, age, number of requests, environmental conditions, etc), the degradation symptoms, the relation between the degradation observation and the appearance of other failure modes, the effects of preventive and corrective maintenance activities, and the planning and execution of maintenance actions,

e. to consider production goals, the safety, health and environment objectives, the maintenance costs and penalties for lost production,

f. to represent the dynamic interactions among the system elements which affect the system behaviour and its maintenance,

g. to characterize, represent and propagate epistemic uncertainties

Some of the advantages of BNs in maintenance optimization strategies are that they allow performing the following aspects: $a$ (Weber et al., 2001), $b$ and $f$ (Weber \& Jouffe, 2006 and Iung et al. 2005), but on the other hand this is a recent field, so there are still missing some issues to be solved such as the points $c, d$, $e$ and $g$.

\section{BAYESIAN NETWORKS MODELING}

This section corresponds to the bibliography related to the comparison between $\mathrm{BN}$ and the two classical methods of dependability evaluation: fault trees and Markov Chains.

\subsection{Fault trees}

The use of fault trees is based on a hypothesis: a Boolean representation for the events. The fault trees are a method solved by the binary decision diagrams (BDD), which enable an exact calculation, considering dependencies between the branches due to redundancy of elementary events not factorized. However, it is necessary to respect the hypothesis of elementary events independency. When multiples failures can potentially affect the components, the model needs a representation of multiple states variables. Thus, the fault trees are not suitable (IEC61025, 2006).

Castillo et al. (1997), Portinale \& Bobbio (1999) and Bobbio et al. (2001) present a relevant contribution in which they explain how fault trees can be translated to $\mathrm{BN}$, maintaining its Boolean nature. So, it is possible to represent fault trees as $\mathrm{BN}$, but the reciprocity is not true. The BNs enable the utilization of modal logic with an unlimited number of modalities and, they make possible and easier the treatment of dependencies based on a DAG (Bouissou \& Pourret, 2003).

BNs can also represent reliability diagrams. The initial work on this area is presented by Torres-Toledano and Sucar 
(1998) who explain the conversion from one representation to the other.

Recently, some papers deal with the link between the new modeling techniques as dynamic fault trees and BNs, for example the articles written by Boudali \& Dugan (2005a, 2006). In these articles, it is proved the equivalence between dynamic fault trees and BN. Dugan and Boudali proposed to include the temporal notion on the variables. This technique requires the $\mathrm{BN}$ modeling with continuous variables.

There is a way to model the dynamic notion by BNs; also there are several techniques called dynamic fault trees. For instance, the publication by Montani et al. (2006) present the transformation of a dynamic fault tree into a $\mathrm{BN}$, with a representation of a DBN discrete of 2 time slices (2TBN).

\subsection{Markov Chains}

A stochastic process can be represented through a group states' description and their transition rate between states. According to the hypotheses assumed for the state transition specifications, the process is markovian, semi markovian or non-markovian. The representation of the state space is identified on the dependability specialized literature (Aven \& Jensen, 1999), (Ansell \& Phillips, 1994), as well as the industrial standards IEC61511, (IEC61511, 2004). This method is suitable for the reliability and availability system studies and it allows analyzing the exact failure probability even when there are dependences between components. The main drawback of Markov Chains method is combinatory explosion of states' number that leads to an unreadable model when studying real industrial systems (De Souza \& Ochoa, 1992).

DBNs enable to represent Markov Chains The first contributors on DBN application to the reliability and availability of systems are Welch \& Thelen (2000). Later, Weber \& Jouffe (2003) have shown the factorization possibility of a markovian model by DBN. The factorization permits to reduce the model complexity and open the possibility to model real systems.

One of the main contributions is a DBNs representation of non homogeneous markovian processes when using changeable parameters through the time (Weber et al., 2004), (Ben Salem et al., 2006). Additionally, Weber et al. (2004) have formalized the inclusion of exogenous variables representing events (maintenance actions, production level, environment conditions) in a degradation process by using a process called MSM (Markov Switching Model), or IOHMM (Input-Output Hidden Markov Model). The originality of the proposed approach is to formalize a component's degradation process and its interaction with the environment by an IO-HMM. The models of these processes, interacting with the environment, can be integrated in a system's global model formalized by an object oriented dynamic bayesian network (OODBN) (Weber et al., 2006).

\section{CONCLUSSION}

The research work and application of Bayesian Networks in risk analysis, dependability, and maintenance have shown an important growing trend since 2000. Especially in dependability nowadays there have been about 30 articles per year, and $800 \%$ of increased publications between 2000 and 2007. This method is particularly suitable to collect and to represent knowledge on uncertain domains, but also enable to perform probabilistic calculus and statistical analyses in an efficient and rigorous manner.

The difference of BNs when comparing with other classical methods is their polyvalence. They allow dealing with issues such as prediction or diagnostic, optimization, data analysis of feedback experience, deviation detection and model updating.

The main issue of the modeling is about the manipulation of the uncertainties within the parameters and the knowledge of the model. The theory of Dempster Shafer proposes an interesting formalism, and the definition of the evidential networks developed by Simon et al. (2009) are very interesting for decision making, considering the imprecision on the utility calculus.

The second interesting issue is to deal with large systems (several hundred of variables), in order to solve the formalism of complex models and to integrate various dimensions (technique, organization, information, decision, finance) correlated with system's behavior in reliability, risk analysis and maintenance fields. The SKOOB project is developing a generic model based on Probabilistic Relational Model (PRM) (Getoor et al., 2007) which enables to have a better understanding of complexity. The network is not defined by a graph but in a language. The inference is performed through partial views of the global model which is actually never built entirely as it is approached in SKOOB project.

The third important issue is the representation of the temporal aspect and/or the continuous variables. For these representations, several inference algorithms exist and are still in development. There efficiency depends on the model complexity (Murphy, 2002)

\section{AKNOWLEDGEMENT}

The authors wish to express their gratitude to the French National Research Agency ANR for the financial support of the Structuring Knowledge with Object Oriented Bayesian nets SKOOB project. Ref. ANR PROJET 07 TLOG 021 (http://skoob.lip6.fr)

\section{REFERENCES}

Ansell J.I., Phillips M.J. (1994). Practical methods for reliability data analysis. Oxford University Press Inc. ISBN 019853664 X.

Arroyo G., Sucar L., Villavicencio A. (1998). Probabilistic temporal reasoning and its application to fossil power plant operation. Expert Systems with Applications. 15, 317-324.

Aven T., Jensen U. (1999). Stochastic Models in Reliability. Applications of mathematics: 41. Edited by I. Karatzas and M. Yor. ISBN 0-387-98633-2, SPIN 10695247, Springer-Verlag, 1999.

Axel B., Helminen A. (2001). A Bayesian belief network for reliability assessment. SAFECOMP 2001, LNCS 2187, 35-45. 
Bai C.G. (2005). Bayesian network based software reliability prediction with an operational profile. Journal of Systems and Software. 77(2), 103-112.

Bai C.G., Hu Q.P., Xie M., Ng S.H. (2005). Software failure prediction based on a Markov Bayesian network model. Journal of Systems and Software. 74(3), 275-282.

Bayraktarli Y., Ulfkjaer J., Yazgan U., Faber M. (2005). On the application of bayesian probabilistic networks for earthquake risk management. 9th International Conference on Structural Safety and Reliability (ICOSSAR 05), Rome, June 20-23.

Ben Salem A., Muller A., Weber P. (2006). Dynamic Bayesian Networks in system reliability analysis. 6th IFAC Symposium on Fault Detection, Supervision and Safety of technical processes, 481-486.

Bobbio A., Portinale L., Minichino M., Ciancamerla E. (2001). Improving the analysis of dependable systems by mapping fault trees into Bayesian networks. Reliability Engineering \& System Safety. 71(3), 249-260.

Boudali H., Dugan J.B. (2006). A continuous-time Bayesian network reliability modeling and analysis framework. IEEE Transaction on Reliability. 55(1), 8697.

Boudali H., Dugan J.B. (2005b). A discrete-time Bayesian network reliability modeling and analysis framework. Reliability Engineering \& System Safety. 87(3), 337-349.

Boudali H., Dugan J.B. (2005a). A new Bayesian network approach to solve dynamic fault trees. IEEE Reliability and Maintainability Symposium. 451-456, January 24-27.

Bouissou M. and Pourret O. (2003). A Bayesian belief network based method for performance evaluation and troubleshooting of multistate systems. Int J Reliab, Qual Saf Eng 10 (4), pp. 407-416.

Bouissou M., Martin F., Ourghanlian A. (1999). Assessment of a Safety Critical System Including Software: a Bayesian Belief Network for Evidence Sources. Reliability and Maintainability Symposium (RAMS‘99). Washington, January 1999.

Bouissou M., Nguyen T. (2002). Decision making based on expert assessments: use of Belief Networks to take into account uncertainty, bias, and weak signals. 13th European Safety and Reliability International Conference (ESREL 2002) Lyon, France, March 2002

Castillo E., Solares C., Gomez P. (1997). Tail uncertainty analysis in complex systems. Artificial Intelligence. 96, 395-419.

Celeux G., Corset F., Lannoy A., Ricard B. (2006) Designing a Bayesian network for preventive maintenance from expert opinions in a rapid and reliable delay. Reliability Engineering \& System Safety. 91(7), 849-856.

Cornalba C., Giudici P. (2004). Statistical models for operational risk management. Physica A. 338, 166-172.

De Melo A.C.V., Sanchez A.J. (2008). Software maintenance project delays prediction using Bayesian Networks. Expert Systems with Applications, In Press, Volume 34, Issue 2. Pages 908-919.

De Souza E., Ochoa P.M. (1992). State space exploration in Markov models. Performance Evaluation Review. 20(1), 152-166.

Duval C., Leger A., Weber P., Levrat E., Iung B., Farret R. (2007). Choice of a risk analysis method for complex socio-technicalsystems. European Safety and Reliability Conference (ESREL 2007). Stavanger, Norway, June 2007.

Embrey D. (2002). Using influence diagrams to analyse and predict failures in safety critical systems. 23rd ESReDA Seminar - Decision Analysis: Methodology and Applications for Safety of Transportation and Process Industries. Delft, The Netherlands, November 2002.

Friis-Hansen P. and Simonsen B.C. (2002). GRACAT: software for grounding and collision risk analysis, Marine Structure 15, pp. 383-401.

Getoor L., Friedman N., Koller D., Pfeffer A., and Taskar B. (2007). Probabilistic Relational Models. In L. Getoor and B. Taskar, editors, Introduction to Statistical Relational Learning, pp. 139-144. MIT Press. USA.

Gulvanessian H., Holicky M. (2001). Determination of actions due to fire: recent developments in Bayesian risk assessment of structures under fire. Building Research Establishment, Garston, Watford, UK, Klokner Institute, Prague, Czech Republic.

Helminen A., Pulkkinen U. (2003). Reliability assessment using Bayesian network - Case study on quantitative reliability estimation of a software-based motor protection relay. VTT Industrial Systems. STUK-YTO-TR 198, Helsinki, pp 28.

Hudson L., Ware B., Laskey K., and Mahoney S. (2002). An application of bayesian networks to antiterrorism risk management for military planners. Technical Report, Digital Sandbox, Inc.

IEC61511. (2004). Functional safety - Safety instrumented systems for the process industry sector, Geneva, IEC.

IEC61025. (2006). Fault tree analysis (FTA). Geneva, IEC.

Iung B., Veron M., Suhner M. and Muller A. (2005). Integration of maintenance strategies into prognosis process to decision making aid on system operation. Annals of the CIRP. 54 (1), pp. 5-8.

Jensen F.V. (1996). An Introduction to Bayesian Networks Editions UCL Press. London, UK.

Kang C.W., Golay M.W. (1999). A Bayesian belief network-based advisory system for operational availability focused diagnosis of complex nuclear power systems. Expert Systems with Applications. 17, 21-32.
Kim M.C, Seong P.H., Hollnagel E. (2006). A probabilistic approach for determining the control mode in CREAM. Reliability Engineering \& System Safety. 91(2), 191-199.

Kim M.C., Seong P.H. (2006). A computational method for probabilistic safety assessment of I\&C systems and human operators in nuclear power plants. Reliability Engineering \& System Safety. 91(5), 580-593.

Langseth H., Portinale L. (2007). Bayesian networks in reliability. Reliability Engineering \& System Safety. 92(1), 92-108.

Lee C. and Lee K.J. (2006). Application of Bayesian network to the probabilistic risk assessment of nuclear waste disposal. Reliability Engineering and System Safety. 91, pp. 515-532.

Léger A., Farret R., Duval C., Levrat E., Weber P., Iung B. (2008). A safety barriers-based approach for the risk analysis of socio-technical systems, 17th IFAC World Congress - 17th IFAC World Congress, Republic of Korea.

Mahadevan S., Zhang R., Smith N. (2001). Bayesian networks for system reliability reassessment. Structural Safety. 23(3), 231- 251.

Montani S., Portinale L., Bobbio A., Varesio M., Codetta-Raiteri D. (2006). A tool for automatically translating Dynamic Fault Trees into Dynamic Bayesian Networks. Reliability and Maintainability Symposium (RAMS 2006), 434-441.

Muller A., Suhner M-C., Iung B. (2008). Formalisation of a new prognosis model for supporting proactive maintenance implementation on industrial system. Reliability Engineering \& System Safety. In Press, 93(2) 234-253.

Murphy K. (2002). Dynamic Bayesian Networks: Representation, Inference and Learning. Phd. University of California, Berkeley, USA.

Neil M., Tailor M., Marquez D., Fenton N., Hearty P. (2008). Modeling dependable systems using hybrid Bayesian networks. Reliability Engineering \& System Safety. Volume 93, Issue 7, Pages 933-939.

Norrington L., Quigley J., Russel A., Van der Meer R. (2007). Modeling the reliability of search and rescue operations with Bayesian Belief Networks. Reliability Engineering \& System Safety. Volume 93, Issue 7, Pages 940-949.

Pearl J. (1988). Probabilistic reasoning in intelligent systems: networks of plausible inference. Morgan Kaufmann Publishers Inc. San Francisco, USA. Portinale L, Bobbio A. (1999). Bayesian networks for dependability analysis: an application to digital control reliability. In: Proceedings of the fifteenth conference on uncertainty in artificial intelligence. San Francisco, CA: Morgan Kaufmann Publishers; p. 551-8.

Simon C., Weber P., Evsukoff A. (2008). Bayesian networks inference algorithm to implement Dempster Shafer theory in reliability analysis. Reliability Engineering and System Safety. Vol. 93, n 7, p. 950-963.

Straub D. (2005). Natural hazards risk assessment using Bayesian networks. 9th International Conference on Structural Safety and Reliability (ICOSSAR 05), Rome, Italy, June 19-23.

Trucco P., Cagno E., Ruggeri F., Grande O. (2008). A Bayesian Belief Network modelling of organisational factors en risk analysis: A case study in maritime. Reliability Engineering \& System Safety. Volume 93, Issue 6, Pages 845-856.

Weber P., Jouffe L. (2003). Reliability modeling with Dynamic Bayesian Networks. Reliability Engineering \& System Safety. Volume 91, Issue 2, Pages 149-162.

Weber P., Jouffe L. (2006). Complex system reliability modeling with Dynamic Object Oriented Bayesian Networks (DOOBN). Reliability Engineering \& System Safety. Volume 91, Issue 2, 149-162.

Weber P., Munteanu P., Jouffe L. (2004). Dynamic Bayesian Networks modelling the dependability of systems with degradations and exogenous constraints. 11th IFAC Symposium on Information Control Problems in Manufacturing (INCOM'04). Salvador-Bahia, Brazil, April 5-7th.

Weber P., Suhner M.-C., Iung B. (2001). System approach-based Bayesian Network to aid maintenance of manufacturing process. 6th IFAC Symposium on Cost Oriented Automation, Low Cost Automation. Berlin, Germany, 33-39, October 8-9.

Welch R. L. (2001). BOLARR: A software product for Bayesian online assessment of reliability and risk, NSF SBIR award 1761391, Phase I Final Report, Gensym Corporation.

Welch R., Thelen T. (2000). Dynamic reliability analysis in an operational context: the Bayesian network perspective, In Dynamic reliability: future directions, Edited by: C. Smidts, J. Devooght and P.E. Labeau, ISBN 0 96526693 1, Maryland, USA.

Wilson A.G., Huzurbazar A.V. (2006). Bayesian networks for multilevel system reliability. Reliability Engineering \& System Safety. Volume 92, Issue 10, pp 1413-1420.

Zio E. (2009). Reliability engineering: Old problems and new challenges. Reliability Engineering \& System Safety. Volume 94, pp. 125-141. 